\title{
Tossing and turning with degenerative arthropathy: an assessment of poor sleep quality in knee osteoarthritis
}

\section{Richard Oluyinka Akintayo ${ }^{1}$, Abubakar Yerima ${ }^{2}$, Courage Uhunmwangho ${ }^{3}$, Hakeem Olaosebikan ${ }^{4}$, Akpabio Akanimo Akpabio ${ }^{5}$}

${ }^{1}$ Department of Medicine, University of Ilorin Teaching Hospital, Ilorin, Nigeria

${ }^{2}$ Department of Medicine, University of Maiduguri Teaching Hospital, Maiduguri, Nigeria

${ }^{3}$ Department of Medicine, Jos University Teaching Hospital, Jos, Nigeria

${ }^{4}$ Department of Medicine, Lagos State University Teaching Hospital, Lagos, Nigeria

${ }^{5}$ Department of Internal Medicine, University of Uyo Teaching Hospital, Uyo, Nigeria

\begin{abstract}
Objectives: To determine the frequency and predictors of sleep abnormalities among patients with knee osteoarthritis (OA) in Nigeria.

Material and methods: A multi-centre, hospital-based, cross-sectional study, involving 250 knee OA patients. Consenting patients 18 years and above, who satisfied the American College of Rheumatology (ACR) criteria for knee OA were recruited from five Nigerian tertiary centres over 3 months. An interviewer-administered questionnaire was used to collect demographic and relevant clinical information. The Pittsburgh Sleep Quality Index (PSQI) was used to assess sleep quality with scores $\geq 5$ indicating poor sleep. Other variables assessed were pain, depression, functional class and family functioning. Data were summarized using appropriate measures of central tendency and dispersion. Multiple logistic regression analysis was done to identify predictors of poor sleep. Analysis was done using SPSS version 21.0 with $p<0.05$ considered significant. Study approval was obtained from the ethical committees of each of the study sites.

Results: Participants included 209 females (83.6\%) with mean age $59.9 \pm 10.6$ years. One hundred and forty-one participants (56.4\%) had PSQI scores $\geq 5$ (poor sleep). This was significantly associated with depression $(p<0.001)$, level of education $(p=0.001)$, higher pain scores $(p<0.001)$, body mass index $(p=0.040)$, medial knee OA $(p=0.032)$ and patello-femoral OA $(p=0.002)$. Higher level of education, worse depression scores and higher WOMAC pain scores were the best predictors of poor sleep quality.

Conclusion: Sleep quality was poor in over half of our knee OA patients and best predicted by depression, pain and level of education. Regular sleep quality assessment for knee OA patients is recommended.
\end{abstract}

Key words: sleep quality, knee osteoarthritis, pain, depression.

\section{Introduction}

Osteoarthritis (OA) is the most common form of arthritis and a leading cause of years lived with disability (YLD) and reduced quality of life [1, 2]. Osteoarthritis commonly affects the elderly and the commonest site affected is the knee; major symptoms are pain, stiffness and activity limitation leading to poor quality of life and psychosocial comorbidities [3].

Sleep disturbance is one of the comorbidities frequently associated with knee OA. Previous reports indicate that $28 \%$ to $80 \%$ of patients with knee OA have sleep disturbance [4]. The Johnston County Osteoarthri-

Address for correspondence:

Akpabio Akanimo Akpabio, Department of Internal Medicine, University of Uyo Teaching Hospital, 520261 Uyo, Nigeria,

e-mail: petersakpabio@yahoo.com

Submitted: 21.07.2019; Accepted: 20.08.2019 
tis Project on sleep and arthritis reported that $71 \%$ of US patients with knee OA had sleep abnormalities [5]. This high frequency of abnormal sleep among knee OA patients is not surprising given that pain, the commonest symptom of osteoarthritis, largely influences the sleep quality of individuals [6].

The relationship between sleep and pain is complex and multidimensional. Nocturnal pain reduces sleep quality, leading to difficulty initiating or maintaining sleep, while the un-refreshing nature of poor sleep contributes to pain and fatigue $[7,8]$. Similarly, knee OA patients with poor functional status have been found to have poor sleep quality with resultant fatigue and lower physical and mental function scores [7, 8].

As a disease of the elderly, osteoarthritis affects a group of individuals who require a lot of family support especially regarding activities of daily living. Previous reports have shown a relationship between family function and subjective well-being, beyond the pain and fatigue experienced by patients [9]. Sleep disturbance is also frequently associated with depression. A study by Parmelee et al. [10] showed that sleep was independently associated with pain and depression with the sleeppain relationship mediated by depressive symptoms.

To the best of our knowledge, multicentre studies on the impact of clinical features of knee OA on sleep quality among Nigerians are lacking. The main aim of this study is to determine the impact of pain, functional status, family function and depression on the sleep quality of patients with knee osteoarthritis.

\section{Material and methods \\ Study design}

We conducted a multi-centre, hospital-based crosssectional descriptive and analytic study at the Rheumatology clinics of five tertiary institutions in Nigeria (University of Maiduguri Teaching Hospital, Jos University Teaching Hospital, University of Ilorin Teaching Hospital, Lagos State University Teaching Hospital, and University of Uyo Teaching Hospital) all spread across the northern, middle and southern parts of the country.

\section{Study population}

Individuals aged 18 years and above, satisfying the American College of Rheumatology (ACR) clinical classification criteria for knee OA [11], were recruited over a period of 3 months.

\section{Sample size determination}

Sample size was obtained using Fisher's statistical formula for estimating minimum sample size in descrip- tive health studies for population sizes greater than 10,000 [12]. Based on the reported knee OA prevalence of $11.5 \%$ determined by Adebusoye et al. in Ibadan, Southwest Nigeria [13], the calculated sample size for the study was estimated to be 156 . However, to increase the power of the study, we recruited a total of $250 \mathrm{pa}$ tients at a rate of 50 patients per centre.

\section{Sampling method}

Patients were recruited using a systematic random sampling method in which every other patient with knee OA was recruited. Prior communication with the co-investigators representing each of the participating hospitals revealed that a median of 100 patients with knee OA were seen over a period of three months. With a recruitment plan of 50 participants per centre, a sampling interval of 2 was chosen. The first patient seen each day meeting the inclusion criteria for the study was chosen followed by the $3^{\text {rd }}$ patient and so on. If an individual did not satisfy the inclusion criteria, or the person refused to participate, the next individual was recruited. Patients' folders were labelled and a research register was kept to avoid double sampling. The procedure was repeated each day until the sample size was attained.

\section{Inclusion and exclusion criteria}

Consenting patients aged 18 and older who satisfied the ACR clinical classification criteria for knee OA served as inclusion criteria. Patients with a history of previous knee surgery, known mental illness, previous diagnosis of insomnia, inflammatory arthritis, systemic infection, or malignancy and other symptomatic chronic diseases, e.g. diabetes mellitus, chronic liver disease, chronic obstructive lung disease, were excluded from the study.

\section{Data collection}

Informed verbal and written consent was obtained from all participants while a semi-structured interviewer-administered questionnaire was used to collect demographic and relevant clinical information. Pain severity was assessed using a 0-10 numerical rating scale (NRS), with 0 representing no pain and 10 being maximum pain experienced. A score of 4 or less was considered mild pain, 5-7 as moderate and 8 and above as severe pain. The WOMAC (Likert) pain subscale was also used to grade pain intensity. We considered cut-off points of less than 5, 6-10 and 11 to 20 as mild, moderate and severe pain respectively.

Individuals were grouped into four functional classes using the Steinbrocker functional classification [14]. Each knee was examined separately for the presence or absence of deformities, effusion, joint line tenderness 
and crepitus. The range of motion (ROM) and alignment of the knee joint were measured using an International Standard Goniometer. Quadriceps strength was graded using the Medical Research Council grading scale. The weight to the nearest $0.01 \mathrm{~kg}$ and height to the nearest $0.01 \mathrm{~m}$ were measured using a Seca electronic weighing/ height scale (Seca Deutschland, Hamburg Germany) using standard techniques. Body mass index (BMI) was calculated using the formula, weight $(\mathrm{kg}) /$ height $^{2}(\mathrm{~m})$. For the purpose of our analysis we grouped patients as not obese $\left(\mathrm{BMI}<30 \mathrm{~kg} / \mathrm{m}^{2}\right)$ or obese $\left(\mathrm{BMI} \geq 30.0 \mathrm{~kg} / \mathrm{m}^{2}\right)$.

Blood pressure (BP) was measured with a mercury sphygmomanometer (Accosson, England) using standard techniques [15]. Standard antero-posterior (AP) and lateral semi-flexed radiographs of both knees in weight bearing position were taken by qualified radiographers of the radiology department of the hospitals. The radiographs were interpreted and graded by both the radiologists and the principal investigators using the Kellgren and Lawrence $(K L)$ criteria [16]. The higher $K L$ grade of the knee was used for the final analysis.

The sleep quality of respondents was assessed using the Pittsburgh Sleep Quality Index (PSQI) [17]. The 19-item questionnaire generates seven components: sleep quality, sleep latency, sleep duration, habitual sleep efficiency, sleep disturbances, use of sleep medications and daytime dysfunction. The global score ranges from 0 to 21 , with higher scores indicative of poorer subjective sleep quality. The PSQI has been validated in Nigeria by Aloba et al. [18] with the best cut-off score set at 5. We dichotomized patients into two groups, scores less than 5 (no sleep abnormality) and score $\geq 5$ (poor sleep quality).

Family functioning was assessed by the Family APGAR (Adaptation, Partnership, Growth, Affection, and Resolve) questionnaire. The measure consists of five parameters of family function: Adaptability, Partnership, Growth, Affection and Resolve. The instrument has a minimum score of 0 and maximum score of 10 . Family APGAR scores are interpreted as follows: 7-10 = highly functional family; 4-6 = moderately dysfunctional family; 0-3 = severely dysfunctional family. The correlation of the instrument with the previously validated instrument (Pless-Satterwhite index) is 0.80 and the correlation with a clinical report is 0.64 [19]. Family APGAR has been validated and used for previous studies in Nigeria [20].

Depression was assessed using the Patient Health Questionnaire (PHQ-9) [21]. It has a minimum score of 0 and maximum score of 27 . Component scores are rated 0 to 3. The interpretation of the total scores is as follows: 0-4 = no depression; 5-9 = mild depression; 10-14 = moderate depression; $15-19=$ moderately severe depression; $20-27$ = severe depression. It has been validated for use in Nigeria, having a good concurrent validity with the Beck Depression Inventory (BDI) $(r=0.84, p<0.001)$ and good one month test-retest reliability $(r=0.84$, $p<0.001$ ) [22]. The sensitivity and specificity with 5 as the cut-off score for minor depressive disorder is 0.989 and 0.973 respectively, and 0.846 and 0.994 respectively with 10 as the cut-off for major depression [22]. PHQ-9 has also been shown to be a valid and reliable tool for assessing depressive disorders and depression severity among patients with knee OA [23]. We dichotomized patients into two groups: $\mathrm{PHQ}-9<5$ (no depression) and PHQ-9 $\geq 5$ (has depression).

\section{Data analysis}

All data obtained were analyzed using SPSS version 21.0 by IBM USA, Armonk, NY 10504. Demographic and clinical data were summarized using frequencies, percentages and proportions. Normality testing was performed using Shapiro-Wilk statistics. Means of normally distributed continuous variables of participants with poor sleep quality and those without poor sleep quality were compared using the t-test. Tests of association between poor sleep quality and each of the determinants under consideration (pain scores, functional status, family functioning, $\mathrm{KL}$ grades and depression) were done using the chi-square test with Fisher's exact test used where frequencies were $<5$. Pearson's and Spearman's correlation was used to determine correlations between sleep quality and the determinants under consideration. Predictors of poor sleep in patients with knee OA were determined using multiple logistic regression analysis with PSQI score $<5$ and $\geq 5$ as a binary outcome. A $p$-value $<0.05$ was considered statistically significant for all tests.

The study was approved by the Ethical Review Committee of each of the study centres.

\section{Results \\ Demographic characteristics}

The 250 participants comprised 209 (83.6\%) females and 41 (16.4\%) males. One hundred and forty-one (56.4\%) had PSQI scores $\geq 5$, indicative of poor sleep quality. Their mean age was 59.90 \pm 10.62 years, and 97 (38.8\%) were 65 years or older. There was no significant difference in mean ages of those with and without poor sleep $(p=0.353)$. The majority (74.4\%) had a formal education, 185 (74\%) were married, 66 (26.4\%) were unemployed. The mean BMI was $30.82 \pm 5.48$. Patients with poor sleep had higher BMI compared to those without poor sleep $(p=0.04)$. Poor sleep was significantly associated with level of education and smoking $(p=0.001$ and $p=0.019$ respectively). Table I shows the socio-demographic distribution of the patients. 
Table I. Socio-demographic characteristics of participants

\begin{tabular}{|c|c|c|c|}
\hline Variable & $\begin{array}{c}\mathrm{PSQI} \leq 4 \\
n(\%)= \\
109(43.6)\end{array}$ & $\begin{array}{c}\text { PSQI } \geq 5 \\
n(\%)=141 \\
(56.4)\end{array}$ & $p$-value \\
\hline Age & $60.61 \pm 10.29$ & $59.35 \pm 10.88$ & $0.353^{*}$ \\
\hline Gender & & & $0.305^{\#}$ \\
\hline Male & $21(19.3)$ & $20(14.2)$ & \\
\hline Female & $88(80.7)$ & $121(85.8)$ & \\
\hline Level of education & & & $0.001^{\#}$ \\
\hline None & $27(24.8)$ & $19(13.5)$ & \\
\hline Primary & $15(13.8)$ & $28(19.9)$ & \\
\hline Secondary & $19(17.4)$ & $26(18.4)$ & \\
\hline Tertiary & $40(36.7)$ & $34(24.1)$ & \\
\hline Postgraduate & $6(5.5)$ & $18(12.8)$ & \\
\hline Others & $2(1.8)$ & $16(11.3)$ & \\
\hline Marital status & & & $0.316^{\#}$ \\
\hline Single & $3(2.8)$ & $2(1.4)$ & \\
\hline Married & $74(67.9)$ & $111(78.7)$ & \\
\hline Separated & $1(0.9)$ & $2(1.4)$ & \\
\hline Divorced & $3(2.8)$ & $2(1.4)$ & \\
\hline Widowed & $27(24.8)$ & $23(16.3)$ & \\
\hline Occupation & & & $0.518^{\#}$ \\
\hline Business & $23(21.1)$ & $32(22.7)$ & \\
\hline Civil servant & 19 (17.4) & $26(18.4)$ & \\
\hline Others & $15(13.8)$ & $20(14.2)$ & \\
\hline Retired & $24(22.0)$ & $28(19.9)$ & \\
\hline Unemployed & $28(25.7)$ & $35(24.8)$ & \\
\hline Ethnic group & & & $0.137^{\#}$ \\
\hline Hausa & $13(11.9)$ & $7(5.0)$ & \\
\hline Igbo & $8(7.3)$ & $11(7.8)$ & \\
\hline Yoruba & $41(37.6)$ & $47(33.3)$ & \\
\hline Others & $47(43.1)$ & $76(53.9)$ & \\
\hline Diabetes mellitus & & & $0.423^{\#}$ \\
\hline Yes & $16(14.7)$ & $23(16.3)$ & \\
\hline No & $93(85.3)$ & $118(83.7)$ & \\
\hline Hypertension & & & $0.861^{\#}$ \\
\hline Yes & $74(67.9)$ & $88(62.4)$ & \\
\hline No & $35(32.1)$ & $53(37.6)$ & \\
\hline Height & $1.63 \pm 0.08$ & $1.61 \pm 0.07$ & $0.124^{*}$ \\
\hline Weight & $79.37 \pm 12.72$ & $82.04 \pm 17.23$ & $0.176^{*}$ \\
\hline BMI & $30.01 \pm 4.74$ & $31.44 \pm 5.94$ & $0.040^{*}$ \\
\hline
\end{tabular}

\section{Relationship between sleep quality and pain, functional class, APGAR score, depression and Kellgren-Lawrence grade}

The mean \pm SD WOMAC pain score was $8.83 \pm 3.84$. Participants with poor sleep had higher mean pain scores compared to those with good sleep quality (9.60 vs. 7.83, $p<0.001)$. One hundred and eighteen (83.7\%) patients with poor sleep quality had moderate to severe WOMAC pain scores, compared to $66(63.1 \%)$ patients without poor sleep $(p<0.001)$. The proportion of participants with poor sleep increased as pain severity increased ( $p$ for trend < 0.0001). Eighty-four (59.6\%) patients with poor sleep had depression (PHQ-9 score $\geq 5$ ). Poor sleep was strongly associated with depression $(p<0.0001)$ and the proportion of participants with poor sleep quality and depression was higher than those with good sleep quality and depression (59.6\% vs. 19.3\%, $p<0.0001)$. Poor sleep quality was not associated with functional class $(p=0.854)$ or APGAR score $(p=0.137)$ of the participants (Table II).

\section{Radiographic findings}

Most participants (97.2\%) had KL grade II and above. One hundred and eighty-six (74.6\%) had medial knee OA, 156 (62.4\%) had lateral knee OA, while 149 (59.6\%) had patello-femoral knee OA. The proportion of participants with a PSQI score $\geq 5$ (poor sleep quality) was higher among individuals with medial and patello-femoral knee OA compared to those with a PSQI score $\leq 4$ ( $p=0.024$ and $p=0.002$ respectively). There was no significant association between lateral knee $\mathrm{OA}, \mathrm{KL}$ grade and poor sleep ( $p=0.103$ and $p=0.333$ respectively), as shown in Table III.

\section{Predictors of poor sleep quality}

After analyzing variables significantly associated with PSQI scores using multiple logistic regression, higher PHQ-9 scores, WOMAC pain scores and higher level of education were found to be the best predictors of poor sleep quality among the participants $(p<0.001, p=0.035$ and $p=0.013$ respectively). Participants with depression and knee OA were 1.27 times more likely to have poor sleep than those without depression, while those with higher WOMAC pain scores were 1.11 times more likely to have poor sleep compared to those with lower pain scores (Table IV).

\section{Discussion}

Our study revealed that poor sleep quality is common among patients with knee OA and it is strongly associated with depression, moderate to severe knee pain, 
Table II. Association between sleep quality and pain, depression, functional class, APGAR score, and Kellgren and Lawrence grade

\begin{tabular}{|c|c|c|c|c|}
\hline Variable & $\begin{array}{c}\mathrm{PSQI} \leq 4 \\
n(\%)=109(43.6)\end{array}$ & $\begin{array}{c}\text { PSQI } \geq 5 \\
n(\%)=141(56.4)\end{array}$ & $p$-value* & $p$ for trend ${ }^{* *}$ \\
\hline WOMAC pain & & & & $<0.0001$ \\
\hline Mean \pm SD & $7.83 \pm 3.91$ & $9.60 \pm 3.61$ & $<0.001^{\#}$ & \\
\hline Mild $(\leq 5)$ & $43(65.2)$ & $23(34.8)$ & $<0.001$ & \\
\hline Moderate (6-10) & $35(38.0)$ & $57(62.0)$ & & \\
\hline Severe (11-20) & $31(33.7)$ & $61(66.3)$ & & \\
\hline PHQ-9 & & & $<0.001$ & \\
\hline Score $\leq 4$ & $88(80.7)$ & $57(40.4)$ & & \\
\hline Score $\geq 5$ & $21(19.3)$ & $84(59.6)$ & & \\
\hline Functional class & & & 0.854 & \\
\hline Class I & $23(21.1)$ & $35(24.8)$ & & \\
\hline Class II & $60(55.0)$ & $70(49.6)$ & & \\
\hline Class III & $23(21.1)$ & $32(22.7)$ & & \\
\hline Class IV & $3(2.8)$ & $4(2.8)$ & & \\
\hline APGAR score & & & 0.137 & 0.052 \\
\hline $0-3$ & $2(18.2)$ & $9(81.8)$ & & \\
\hline $4-6$ & $15(37.5)$ & $25(62.5)$ & & \\
\hline $7-10$ & $92(46.2)$ & 107 (53.8) & & \\
\hline KL grade & & & 0.333 & 0.475 \\
\hline Grade I & $1(14.3)$ & $6(85.7)$ & & \\
\hline Grade II & $25(41.0)$ & $36(59.0)$ & & \\
\hline Grade III & $51(48.1)$ & 55 (51.9) & & \\
\hline Grade IV & $32(42.1)$ & $44(57.9)$ & & \\
\hline
\end{tabular}

PSQI - Pittsburgh Sleep Quality Index, ${ }^{*}-\chi^{2}$ test, ${ }^{* *}-\chi^{2}$ test for trend, SD - standard deviation, ${ }^{*}-$ Student's t-test, WOMAC - Western Ontario and McMaster Universities index, PHQ-9 - Patient Health Questionnaire score-9, APGAR - Adaptation, Partnership, Growth, Affection, and Resolve, KL - Kellgren-Lawrence.

level of education, obesity as well as medial and patellofemoral knee OA. Level of depression and pain scores were the best predictors of poor sleep among our participants. Sleep abnormalities are fairly common among patients with knee OA. Previous studies have reported frequencies of 11.6 to $71 \%$ of insomnia among patients with arthritis $[5,6]$. This large variation in frequency is due to differences in sleep assessment tools used, populations studied and the region/country involved. The $56.4 \%$ we found in our study is similar to that seen in other studies [4-6].

In our study, there was no difference in sleep quality between males and females, though a study by Quan et al. [24] involving elderly patients in a small city in Korea revealed that more females had insomnia compared to males. This was similar to a report by Arber et al. [25]. These studies involved patients from the general population without particular reference to knee OA, and this might explain the difference.
The relationship between poor sleep and level of education has yielded conflicting results. In a cross-sectional survey of 9,714 individuals by Patel et al. [26], level of education was found to mediate poor sleep in poor individuals only, while Tang et al. in China [27] found that poor sleep was significantly associated with higher level of education. Our study also revealed a strong association between level of education and poor sleep similar to reports by Tang et al. [27] We observed that patients with poor sleep had higher BMI compared to those with good sleep, in agreement with the report by Taylor et al. [4]. Their study involving 300 veterans with insomnia, obstructive sleep apnoea (OSA) and either hip or knee OA showed that obesity was strongly associated with poor sleep and OSA.

Multiple studies [5-8] have all shown a strong association between knee OA pain and poor sleep quality though this relationship is complex. Our study confirmed that pain is strongly related and is a strong predictor of 
Table III. Radiographic findings of participants with good and poor sleep quality

\begin{tabular}{|c|c|c|c|c|}
\hline Variable & $\begin{array}{c}\mathrm{PSQI} \leq 4 \\
n(\%)\end{array}$ & $\begin{array}{c}\mathrm{PSQI} \geq 5 \\
n(\%)\end{array}$ & $p$-value ${ }^{*}$ & $p$-value ${ }^{\star *}$ \\
\hline Medial comp. & & & 0.032 & 0.024 \\
\hline Yes & $73(73.0)$ & $113(85.0)$ & & \\
\hline No & $27(27.0)$ & $20(15.0)$ & & \\
\hline Lateral comp. & & & 0.103 & 0.159 \\
\hline Yes & $59(64.1)$ & $97(74.6)$ & & \\
\hline No & 33 (35.9) & $33(25.4)$ & & \\
\hline Patellofemoral & & & 0.002 & 0.002 \\
\hline Yes & 49 (57.6) & $100(77.5)$ & & \\
\hline No & $36(42.4)$ & $29(22.5)$ & & \\
\hline KL grade & & & 0.333 & $0.475^{\#}$ \\
\hline Grade I & $1(14.3)$ & $6(85.7)$ & & \\
\hline Grade II & 25 (41.0) & $36(59.0)$ & & \\
\hline Grade III & $51(48.1)$ & $55(51.9)$ & & \\
\hline Grade IV & $32(42.1)$ & 44 (57.9) & & \\
\hline
\end{tabular}

PSQI - Pittsburgh Sleep Quality Index, comp. - compartment ${ }^{*}-\chi^{2}$ test, ${ }^{* *}-Z$-test for difference in proportion. ${ }^{*}-\chi^{2}$ test for trend.

Table IV. Predictors of poor sleep quality amongst patients with knee osteoarthritis

\begin{tabular}{|lccccc|}
\hline Variable & B & Wald & $p$-value & OR & Cl \\
\hline PHQ-9 & 0.238 & 16.572 & $<0.001$ & 1.269 & $1.131-1.423$ \\
\hline Level of educatiom & 0.289 & 6.187 & 0.013 & 1.335 & $1.063-1.676$ \\
\hline WOMAC pain score & 0.100 & 4.468 & 0.035 & 1.105 & $1.007-1.212$ \\
\hline PF compartment & -0.689 & 3.470 & 0.062 & 0.502 & $0.223-1.037$ \\
\hline BMI & 0.042 & 1.766 & 0.184 & 1.043 & $0.980-1.102$ \\
\hline KL grade & -0.244 & 1.350 & 0.245 & 0.784 & $0.519-1.182$ \\
\hline
\end{tabular}

OR - odds ratio, Cl - confidence interval, PHQ-9 - Patient Health Questionnaire score-9, PF- patellofemoral, BMI - body mass index, $K L$ - Kellgren-Lawrence.

poor sleep amongst patients with knee OA. Similarly, Louie et al. [6] reported that joint pain and limitation were the main mediators between pain and insomnia. Woolhead et al. [28] found that night pain was associated with poor sleep quality while Sasaki et al. [8] reported that pain severity increased with increasing $\mathrm{KL}$ grade and this was associated with higher rates of poor sleep.

Most of our patients with poor sleep were depressed. Depression has been linked to poor sleep among patients with knee OA in previous studies, similar to what we observed. [4-6, 24]. Parmelee et al. [10] found that the sleep-pain relationship could be explained by depressive symptoms and depression was independently associated with both pain and sleep problems. Our study also identified depression as the best predictor of poor sleep quality, further confirming the association between pain, depression and sleep quality.
Interestingly, we found no association between $\mathrm{KL}$ grade and poor sleep, in contrast with the report by Sasaki et al. [8], though poor sleep was associated with medial and patello-femoral knee OA. This is not surprising in view of the poor correlation between radiographic severity of knee OA and pain, with the latter being a major determinant of poor sleep in our study. Lim et al. [29] reported that as family APGAR scores decreased, the WOMAC pain subscale score increased, indicating that a functional family reduces pain perception by knee OA patients.

We were unable to identify any significant association between poor sleep and family functioning, though the proportion of patients with a severely dysfunctional family and poor sleep was higher than those without poor sleep. Potential limitations of this study include its cross-sectional design, limited study sites and the fact that it was hospital-based. 


\section{Conclusions}

This study has shown that sleep quality is poor among Nigerian knee OA patients and it is largely influenced by depression, pain and higher level of education. Whether treating depression will improve pain and sleep quality remains unanswered. Larger multicentre studies preferably with an intervention arm are hereby recommended to properly assess the impact of pain and depression on sleep quality of knee OA patients.

\section{Acknowledgements}

We acknowledge the contributions of the radiographers and radiologists of all the institutions involved, for their assistance in taking and interpreting the radiographs respectively.

The authors declare no conflict of interest.

\section{References}

1. Lourenço S, Lucas R, Araújo F, et al. Osteoarthritis medical labelling and health-related quality of life in the general population. Health Qual Life Outcomes 2014; 12: 146.

2. Vos T, Flaxman AD, Naghavi M, et al. Years lived with disability (YLDS) for 1160 sequelae of 289 diseases and injuries 19902010: A systematic analysis for the global burden of disease study 2010. Lancet 2012; 380: 2163-2196.

3. Rathbun AM, Yau MS, Shardell M, et al. Depressive symptoms and structural disease progression in knee osteoarthritis: Data from the osteoarthritis initiative. Clin Rheumatol 2017; 36: 155-163.

4. Taylor SS, Hughes JM, Coffman CJ, et al. Prevalence of and characteristics associated with insomnia and obstructive sleep apnea among veterans with knee and hip osteoarthritis. BMC Musculoskelet Disord 2018; 19: 79.

5. Allen KD, Renner JB, Devellis B, et al. Osteoarthritis and sleep: the Johnston County osteoarthritis project. J Rheumatol 2008; 35: 1102-1107.

6. Louie GH, Tektonidou MG, Caban-Martinez AJ, et al. Sleep disturbances in adults with arthritis: prevalence, mediators, and subgroups at greatest risk. Data from the 2007 National Health Interview Survey. Arthritis Care Res 2011; 63: 247-260.

7. Moldofsky H. Sleep and pain. Sleep Med Rev 2001; 5: 385-396.

8. Sasaki E, Tsuda E, Yamamoto Y, et al. Nocturnal knee pain increases with the severity of knee osteoarthritis, disturbing patient sleep quality. Arthritis Care Res (Hoboken) 2014; 66: 1027-1032.

9. Ryan AK, Willits FK. Family ties, physical health, and psychological wellbeing. J Aging Health 2007; 19: 907-920.

10. Parmelee PA, Tighe CA, Dautovich ND. Sleep disturbance in osteoarthritis: linkages with pain, disability, and depressive symptoms. Arthritis Care Res 2015; 67: 358-365.

11. Altman R, Asch E, Bloch D, et al. Development of criteria for the classification and reporting of osteoarthritis. Classification of osteoarthritis of the knee. Diagnostic and Therapeutic Criteria Committee of the American Rheumatism Association. Arthritis Rheum 1986; 29: 1039-1049.
12. Wang D, Bakhai A, Del Buono A, et al. Sample size determination for clinical research. Muscles, Ligaments Tendons J 2013; 3: 116-117.

13. Adebusoye LA, Ogunbode AM, Alonge TO. Magnitude of knee osteoarthritis and associated risk factors among adult patients presenting in a family practice clinic in Nigeria. J Med Trop 2013; 15: $144-150$

14. Hochberg MC, Chang RW, Dwosh I, et al. The American College of Rheumatology 1991 revised criteria for the classification of global functional status in rheumatoid arthritis. Arthritis Rheum 1992; 35: 498-502.

15. Chobanian AV, Bakris GL, Black HR, et al. The Seventh Report of the Joint National Committee on Prevention, Detection, Evaluation, and Treatment of High Blood Pressure: the JNC 7 report. JAMA 2003; 289: 412-419.

16. Yoshida K, Barr RJ, Galea-Soler S, et al. Reproducibility and Diagnostic Accuracy of Kellgren-Lawrence grading for osteoarthritis using radiographs and dual-energy $x$-ray absorptiometry images. J Clin Densitom 2015; 18: 239-244.

17. Buysse DJ, Reynolds CF, Monk TH, et al. The Pittsburgh Sleep Quality Index: A new instrument for psychiatry practice and research. Psychiatry Res 1989; 28: 193-213.

18. Aloba O, Adewuya A, Ola B, et al. Validity of the Pittsburgh sleep quality index (PSQI) among Nigerian University Students. Sleep Med 2007; 8: 266-270.

19. Smilkstein G, Ashworth C, Montano D. Validity and reliability of the family APGAR as a test of family function. J Fam Pract 1982; 15: 303-311.

20. Muyibi AS, Ajayi IO, Irabor AE, et al. Relationship between adolescents' family function with socio-demographic characteristics and behaviour risk factors in a primary care facility. Afr J Prim Health Care Fam Med 2010; 2: 177.

21. Kroenke K, Spitzer RL, Williams JB. The PHQ 9: validity of a brief depression severity measure. J Gen Intern Med 2001; 16: 606-613.

22. Adewuya AO, Ola BA, Afolabi OO. Validity of the patient health questionnaire (PHQ-9) as a screening tool for depression amongst Nigerian university students. J Affect Disord 2006; 96: 89-93.

23. Rosemann T, Backenstrass $M$, Joest $K$, et al. Predictors of depression in a sample of 1,021 primary care patients with osteoarthritis. Arthritis Rheum 2007; 57: 415-422.

24. Quan SA, Li Y, Li WJ, et al. Gender Differences in Sleep Disturbance among Elderly Koreans: Hallym Aging Study. J Korean Med Sci 2016; 31: 1689-1695.

25. Arber S. Gender, marital status and sleep problems in Britain. Przegl Lek 2012; 69: 54-60.

26. Patel NP, Grandner MA, Xie D, et al. "Sleep disparity" in the population: poor sleep quality is strongly associated with poverty and ethnicity. BMC Public Health 2010; 10: 475.

27. Tang J, Liao Y, Kelly BC, et al. Gender and Regional Differences in Sleep Quality and Insomnia: A General Population-based Study in Hunan Province of China. Sci Rep 2017; 7: 43690.

28. Woolhead G, Gooberman-Hill R, Dieppe P, et al. Night pain in hip and knee osteoarthritis: a focus group study. Arthritis Care Res (Hoboken) 2010; 62: 944-949.

29. Lim AT, Manching J, Penserga EG. Correlation between Family APGAR scores and health-related quality of life of Filipino elderly patients with knee osteoarthritis. Int J Rheum Dis 2012; 15: 407-413. 